\title{
Role of Apigenin in Cancer Prevention via the Induction of Apoptosis and Autophagy
}

REVIEW

\author{
Bokyung Sung, Hae Young Chung, Nam Deuk Kim \\ Department of Pharmacy, College of Pharmacy, Molecular Inflammation Research Center for Aging Intervention, Pusan National University, Busan, \\ Korea
}

\begin{abstract}
Apigenin (4',5,7-trihydroxyflavone) is a flavonoid commonly found in many fruits and vegetables such as parsley, chamomile, celery, and kumquats. In the last few decades, recognition of apigenin as a cancer chemopreventive agent has increased. Significant progress has been made in studying the chemopreventive aspects of apigenin both in vitro and in vivo. Several studies have demonstrated that the anticarcinogenic properties of apigenin occur through regulation of cellular response to oxidative stress and DNA damage, suppression of inflammation and angiogenesis, retardation of cell proliferation, and induction of autophagy and apoptosis. One of the most well-recognized mechanisms of apigenin is the capability to promote cell cycle arrest and induction of apoptosis through the p53-related pathway. A further role of apigenin in chemoprevention is the induction of autophagy in several human cancer cell lines. In this review, we discuss the details of apigenin, apoptosis, autophagy, and the role of apigenin in cancer chemoprevention via the induction of apoptosis and autophagy.
\end{abstract}

(J Cancer Prev 2016;21:216-226)

Key Words: Apigenin, Chemoprevention, Apoptosis, Autophagy

\section{INTRODUCTION}

Global changes in fertility and life expectancy have resulted in a rapidly increasing and aging population. As a result, the magnitude of cancer has also grown substantially in every country. In the United States of America, more than 1,685,210 new cancer cases and 595,690 cancer deaths are predicted in 2016. ${ }^{1}$ According to a recent report, the projected global cancer burden will rise from 14.1 million new cancer cases in 2012 to 20 million new cases by 2025 , which indicates population growth and an evolving age distribution, together with other important changes in underlying incidence allied with the prevalence and distribution of risk factors. ${ }^{2}$ A large body of evidence clearly indicates that primary prevention of cancer is an effective way to fight cancer, with between one-third and one-half of cancers being preventable.
Cancer chemoprevention involves the chronic administration of a synthetic, natural, or biological agents, as either an individual drug or dietary supplement, to reduce or delay the occurrence of malignancies. Methodologically, chemoprevention is categorized as primary, secondary, or tertiary. Primary chemoprevention is suitable for the general healthy population who may be at increased risk of disease. Secondary chemoprevention involves administration of agents to reduce the progression of cancer that has already developed in patients with premalignant lesions. Definitions of primary and secondary chemoprevention vary and some groups now combine the two scenarios under the category of "primary chemoprevention". The representative examples of primary chemopreventive agents include dietary phytochemicals and non-steroidal anti-inflammatory drugs. Tertiary chemoprevention aims to prevent the disease recurrence or the development of additional primary disease in individuals who have

Received November 16, 2016, Revised November 28, 2016, Accepted November 29, 2016

Correspondence to: Nam Deuk Kim

Department of Pharmacy, College of Pharmacy, Pusan National University, 2 Busandaehak-ro 63beon-gil, Geumjeong-gu, Busan 46241, Korea Tel: +82-51-510-2801, Fax: +82-51-513-6754, E-mail: nadkim@pusan.ac.kr, ORCID: Nam Deuk Kim, http://orcid.org/0000-0001-9033-9865

Copyright (C) 2016 Korean Society of Cancer Prevention

(c) This is an Open Access article distributed under the terms of the Creative Commons Attribution Non-Commercial License (http://creativecommons.org/licenses/by-nc/4.0) which permits unrestricted non-commercial use, distribution, and reproduction in any medium, provided the original work is properly cited. 
undergone successful treatment of early disease. At the molecular level, cancer chemoprevention is categorized by the disruption of multiple pathways and processes of the three stages of carcinogenesis: initiation, promotion, and progression. Agents that inhibit the initiation stage are termed "blocking agents" because they may act by preventing interactions between mutagenic substance with DNA, which results in mutations that contribute to not only cancer initiation but also to progressive genomic instability and neoplastic transformation. Agents that inhibit promotion and progression are often referred as "suppressing agents" for their ability to perturb the effects of tumor promoters. In recent years, cancer chemoprevention has emerged as a major approach for the reduction of cancer burden. ${ }^{3}$

Phytochemicals are non-nutritive plant chemicals with protective or disease preventive properties. Phytochemicals are one of typical chemopreventive agents as mentioned above, and exist in abundance in fruits and vegetables. The current consensus is that, in general, cancer risk is inversely associated with the consumption of fruits and vegetables. ${ }^{4}$ Polyphenols present in plant-based foods, such as fruits and vegetables, are the most extensively studied group of phytochemicals. Flavonoids are a large subgroup of polyphenols that are present in a wide range of fruits, vegetables, and grains. Abundant evidence speculates that flavonoids may be helpful in the reduction of cancer risk simply by functioning as antioxidants and eliminating free radicals that have been linked to DNA damage and inflammation. Previous case-control studies have shown that intake of total flavonoids, flavonoid subgroups, or individual flavonoids was associated with a reduced risk of lung, gastric, colorectal, breast, ovarian, and endometrial cancers and non-Hodgkin's lymphoma. ${ }^{5-10}$ Interestingly, one case-control study evaluated the association between intake of five common dietary flavonoids (myricetin, kaempferol, quercetin, luteolin, and apigenin) and ovarian cancer risk in 1,141 patients with ovarian cancer and 1,183 frequency-matched control subjects. ${ }^{8}$ There was no clear association between the incidence of ovarian cancer and the total intake of the five flavonoids examined. In analyses of each individual flavonoid, only apigenin intake was associated with decreased cancer risk, and the association was only of borderline significance. Given the promising findings from other studies on the association between dietary flavonoid intake and cancer risk, as well as the evidence of an inverse association with apigenin intake, additional studies of apigenin intake and cancer risk are warranted.

\section{NATURAL SOURCES OF APIGENIN}

Flavonoids (or bioflavonoids) are a class of plant and fungus secondary metabolites. They were discovered in the 1930s by the Hungarian biochemist Rusznyák and Szent-Györgyi. ${ }^{11}$ They isolated a substance from citrus rind, which they called citrin or vitamin $\mathrm{P}$, that contained a mixture of flavonoids. Later, vitamin P was renamed flavonoids. ${ }^{11}$ Since this discovery, scientists have identified more than 7,000 flavonoids and the list of newly-discovered flavonoids continues to grow. ${ }^{12}$

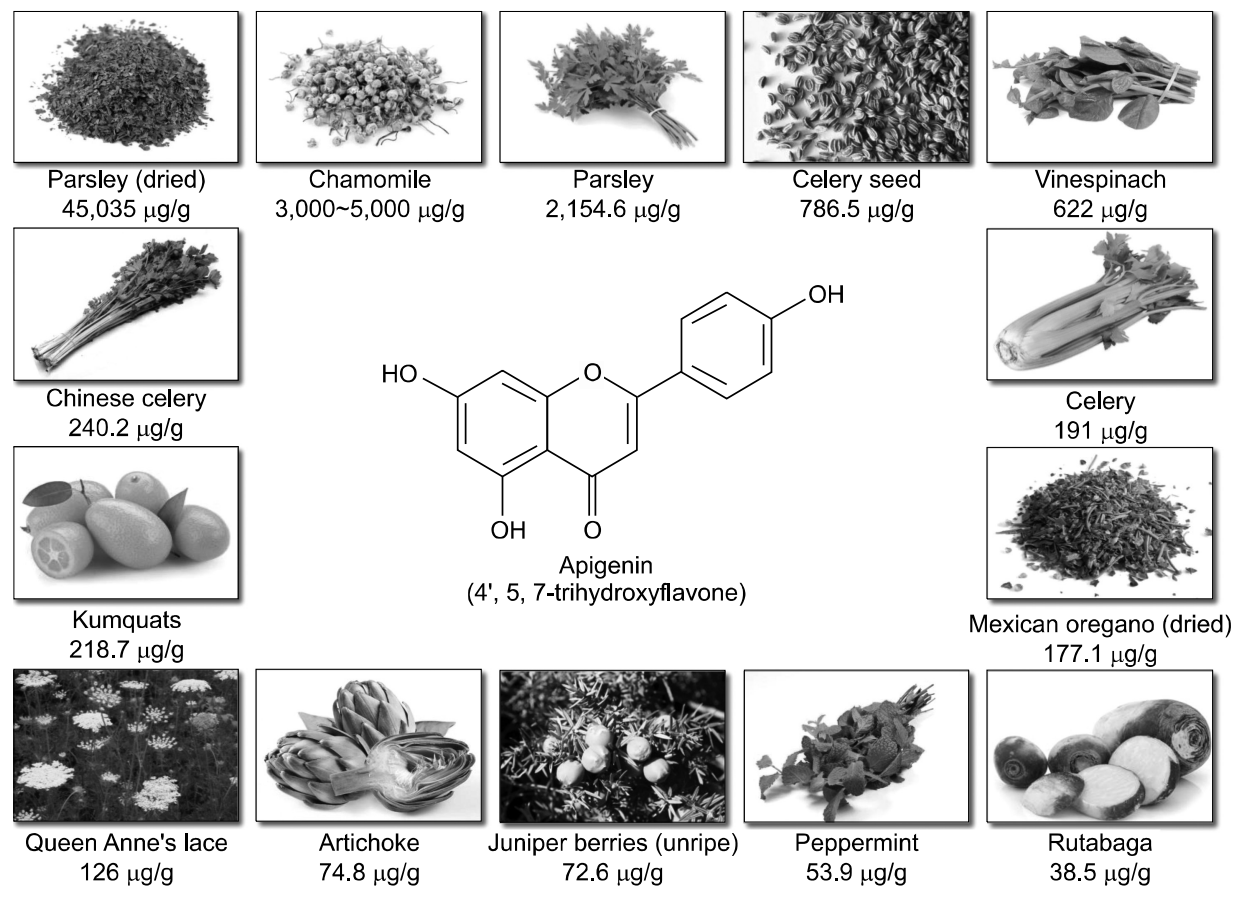

Figure 1. Structure and natural sources of apigenin. Data from US Department of Agriculture (2011; https:// www.ars.usda.gov/ARSUserFiles/8040 0525/Data/Flav/Flav_R03.pdf). 
The compound 4',5,7-trihydroxyflavone is a natural flavone commonly referred to as apigenin. The name "apigenin", like many other flavonoids, is derived from Apiumgenus in Apiaceae (celery, carrot or parsley family, also known as Umbelliferae). The compound has hydroxyl groups at positions C-5 and C-7 of A-ring and C-4' of B-ring, and belongs to a class of flavonoids known as flavones (Fig. 1). The molecular formula of apigenin is $\mathrm{C}_{15} \mathrm{H}_{10} \mathrm{O}_{5}$ and molecular weight is MW 270.24; it is a yellow crystalline powder insoluble in water and soluble in dimethyl sulfoxide and hot ethanol.

Apigenin is regarded as one of the major flavonoids because of its presence and abundance in a variety of natural sources, including fruits and vegetables. Major sources of apigenin include parsley, chamomile, celery, vinespinach, artichokes, and oregano. Among these, dried parsley is the richest source of apigenin, containing $45,035 \mu \mathrm{g} / \mathrm{g}$. Other sources of high apigenin content are chamomile (dried flower), celery seed, vinespinach, and

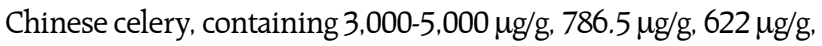
and $240.2 \mu \mathrm{g} / \mathrm{g}$, respectively (Fig. 1). Other than apigenin, various glycosylated derivatives (e.g., apiin and apigetrin) and dimers of apigenin such as amentoflavone ( $3^{\prime}, 8^{\prime \prime}$-biapigenin) have been isolated from natural sources.

\section{ROLE OF APIGENIN IN CANCER PREVENTION}

In the 1980s, Birt et al. ${ }^{13}$ first demonstrated the effective anti-mutagenic and anti-promotive properties of apigenin. Since then, the potential value of apigenin in cancer prevention and treatment has been further supported by extensive research in various animal models of cancer.

The chemopreventive effect of apigenin was explored in at least dozen in vivo studies, which tested doses, administration routes, and treatment frequencies of apigenin. The oral administration of apigenin (20 and $50 \mu \mathrm{g} /$ mice) for 20 weeks reduced tumor volumes and induced complete abolishment of distant organ metastasis in the transgenic adenocarcinoma of a mouse prostate (TRAMP) model. This effect was attributed to the suppression of the phosphoinositide 3-kinase (PI3K)/Akt/ Forkhead box O-signaling pathway. ${ }^{14}$ The same research group also showed that apigenin effectively suppressed prostate cancer progression in TRAMP mice by attenuation of insulin-like growth factor (IGF)-I/IGF binding protein-3 signaling and inhibition of angiogenesis and metastasis. ${ }^{15}$ In addition, a 15 -week period of oral administration of apigenin $(2.5 \mathrm{mg} / \mathrm{kg})$ in hamsters resulted in reduction of tumor volume and incidence, modulation of cell proliferation, apoptosis, inflammation, and angiogenesis markers, and modulation of phase I and II detoxification cascades in a 7,12-dimethyl benz[a]anthracene (DMBA)-induced experimental oral carcinogenesis model ${ }^{16,17}$ The chemoprotective effect of apigenin against oral carcinogenesis was further supported by studies reporting that this flavone lowered tumor incidence in DMBA-induced animal model. ${ }^{18.19}$ Wei et al. ${ }^{20}$ investigated the potential chemopreventive effects of apigenin on murine skin tumorigenesis initiated by DMBA and promoted by 12 -O-tetradecanoylphorbol-13-acetate in SENCAR mice. Their findings revealed that topical application of apigenin $(5$ and $20 \mu \mathrm{mol})$ results in a marked reduction of incidence and number of papillomas as well as carcinomas.

A study by Byun et al. ${ }^{21}$ showed reduction of UVB-induced ear edema and inflammatory mediator COX-2 expression in the skin of SKH-1 hairless mouse, reflecting the potent chemopreventive activity of apigenin against UVB-induced skin inflammation. A topical application of apigenin $(5 \mu \mathrm{M})$ prior to UVB-exposure attenuated the expression of COX-2 and hypoxia inducible factor (HIF)- $1 \alpha$, important mediators of angiogenesis, through modulation of HuR and thrombospondin-1. ${ }^{22}$ Another study showed that apigenin inhibited activation of the UVB-induced mammalian target of rapamycin (mTOR), cell proliferation, and cell cycle progression in mouse skin. The same study also demonstrated that apigenin inhibited UVB-induced mTOR signaling mainly through the activation of AMP-activated protein kinase (AMPK), rather than the suppression of Akt, even though UVB-induced mTOR activation is driven by PI3K/Akt signaling and apigenin is capable of blocking Akt phosphorylation/activation. ${ }^{23}$

Assessment of the chemopreventive potential of apigenin in a colon carcinogenesis model induced by azoxymethane injection in rats demonstrated that dietary intake of this flavone (0.1\%) triggered apoptosis of luminal surface colonocytes and reduced the incidence of aberrant crypt foci, the earliest identifiable lesions in the development and progression of colon cancer, particularly in the tumor initiation phase. ${ }^{24}$ In the same model, subcutaneous injections of apigenin $(0.75$ and $1.5 \mathrm{mg} / \mathrm{kg}$ body weight) significantly decreased the incidence of peritoneal metastasis of intestinal adenocarcinomas. ${ }^{25}$ Similarly, in $\mathrm{APC}^{\mathrm{Min} /+}$ mice, ${ }^{26}$ oral administration of apigenin reduced the number of polyp by the activation of $\mathrm{p} 53$, a tumor suppressor gene. Overall, these results reflect the beneficial effect of apigenin against chemical- and mutation-induced carcinogenesis.

Numerous in vivo examples have suggested that apigenin could suppress tumor growth and metastasis. The oral administration of apigenin (20 and $50 \mu \mathrm{g} /$ mice) decreased the volume 
and wet weight of tumors in nude mice bearing human prostate cancer, without any undesirable side effects. ${ }^{27-30}$ Apigenin intake also decreased serum-IGF-I level and induced apoptosis and cell cycle arrest in tumor xenograft. ${ }^{27,29,31,32}$ Apigenin was shown to interrupt the NF- $\mathrm{KB}$ signaling pathways; this was responsible for the suppression of prostate cancer progression. ${ }^{30}$ Moreover, apigenin significantly decreased the level of Her2/neu, a protein involved in growth factor signaling, which indicated a reduction in breast cancer cell proliferation in apigenin-treated mice. ${ }^{33}$ Apigenin also displayed significant antitumor activity in human colon cell implanted nude mice. ${ }^{34.35}$ The anti-metastatic activity of apigenin has also been reported in an orthotopic colorectal nude mouse model. ${ }^{36}$ Apigenin treatment in mice carrying A549 lung cancer xenografts reduced the tumor volume. This was partially attributed to interruption of the HIF- $1 \alpha$-vascular endothelial growth factor pathway and subsequent suppression of angiogenesis and cell proliferation. ${ }^{37}$ Likewise, the intake of an apigenin in diet $(0.2 \%)$ for six weeks produced significant antitumor activity in an orthotopically implanted nude mouse model of human pancreatic cancer. ${ }^{38}$ Overall, the majority of these studies indicate that apigenin can inhibit tumor initiation, progression, and metastasis in a wide variety of preclinical cancer models.

\section{ROLE OF APIGENIN IN APOPTOSIS}

Programmed cell death (PCD), referring to apoptosis or type I PCD, is one of the most well-characterized types of cell death. PCD is an evolutionally conserved and fundamental process involved in the regulation of various physiological conditions, such as the development and homeostasis of multicellular organisms. Apoptosis is important in cancer biology because PCD is a critical process by which abundant or undesirable cells can be removed. A huge body of evidence demonstrates that deregulation or mutation of apoptosis contributes to the development of numerous pathological conditions, including neurodegenerative diseases, autoimmunity, and cancer. ${ }^{39,40}$ Two core pathways exist to induce apoptosis: the extrinsic [death receptor (DR)] pathway and intrinsic (mitochondrial) pathway. The extrinsic pathway is activated by the binding of DRs (Fas and other similar receptors, such as TNF receptor 1 and its relatives) with their cognate ligands. Upon engaging with their ligands, DRs induce the formation of death-inducing signaling complex by recruitment of caspase- 8 and lead the activation of caspase cascade. The activated caspase- 8 can either directly activate effector caspases such as caspase-3 or indirectly initiate activation of the intrinsic pathway of apoptosis. ${ }^{41}$ The intrinsic pathway is triggered by the release of mitochondrial intermembrane space proteins into the cytosol. In both cases, when a cell is stimulated by either extracellular or intracellular signals, the outer mitochondrial membrane becomes permeable to internal cytochrome $c$, which is then released into the cytosol. The released cytochrome $c$ activates caspases by forming apoptosome, a protein complex composed of cytochrome $c$ and apoptotic peptidase activating factor 1, leading to caspase-9 and subsequently caspase-3 activation and culminating at the conclusion of the extrinsic pathway: apoptosis. $^{42}$

The effects of apigenin on apoptosis have been studied extensively in cell populations of many different cancers. Apigenin has been shown either to directly induce apoptosis or to sensitize cells to other pro-apoptotic stimuli in cancer cell lines of oral, ${ }^{43}$ esophageal, ${ }^{44,45}$ colorectal, $^{26,46-48}$ hepatic, ${ }^{49,50}$ and pancreatic ${ }^{51}$ cancers. Recent work in pancreatic carcinoma has indicated the possibility that apigenin was capable to promote cell cycle arrest and induction of apoptosis through p53-related pathways, even where cancers harbor mutations of the $\mathrm{p} 53$ tumor suppressor. ${ }^{38}$

Apigenin was shown to sensitize human colorectal carcinoma to apoptosis. A comparative study of nine dietary flavonoids was performed to examine their effect on cell growth in HCT116 human colon cancer cells. ${ }^{26}$ Among the tested flavonoids, apigenin was found to be the most potent inhibitor of cell growth. Apigenin also induced apoptosis in other colon cancer cell lines, including HT-29, SW480, and LoVo. ${ }^{26,46}$ The concentrations of apigenin used in these studies have typically been in the 1-90 $\mu \mathrm{mol}$ range and exposure times were 24 to 72 hours. Treatment of these cells with apigenin leads to the induction of the cell cycle inhibitor p21/WAF1, pro-apoptotic protein p53, and nonsteroidal anti-inflammatory drug-activated gene, caspase-3 activation, and DNA fragmentation. ${ }^{26,46}$ Changes in phosphorylation were observed in response to apigenin in HCT116 cells and also found in apigenin-treated tumor tissues from $A P C^{M I N+}$ mice. $^{26}$

\section{ROLE OF APIGENIN IN AUTOPHAGY}

\section{Autophagy}

The term "autophagy" ("self-eating", and derived from the Greek) was coined by De Duve and Wattiaux, ${ }^{52}$ who discovered a process in which the cell digested its own cytoplasmic materials within lysosomes. Nowadays, autophagy is defined as a catabolic membrane-trafficking process that leads to sequestration and degradation of macromolecules within lysosomes. ${ }^{53}$ The last decade has seen a large rise in the study of autophagy due to its 
involvement with various diseases and stresses, as well as normal developmental processes and aging. ${ }^{54,55}$ There are three primary forms of autophagy: microautophagy, macroautophagy, and the mechanistically unrelated process, chaperone-mediated autophagy (CMA). The different forms are characterized by their function and delivery mode of the cargo to lysosomes. ${ }^{53}$ In macroautophagy, proteins are sequestered in double membrane vesicles that form in the cytosol and then fuse with lysosomes to transfer their contents for degradation. ${ }^{56}$ In microautophagy, proteins are engulfed inside vesicles that form directly through the invagination of the lysosomal membrane. A third mode of autophagy, CMA, only occurs in mammalian cells. ${ }^{57}$ CMA differs from the other modes of autophagy in both the way in which cargo proteins are recognized for lysosomal delivery and the way in which these proteins reach the lysosomal lumen. In CMA, internalization of the substrate protein is preceded by its unfolding, a step not required in the other types of autophagy. Despite several recent works that have emphasized the important roles of microautophagy or CMA in tumor growth and progression, macroautophagy is the most studied form; almost all of the research related to autophagy and cancer development, progression, and therapy is centered on macroautophagy.

\section{Autophagy and cancer}

There is growing evidence that the relationship between autophagy and cancer is complex. ${ }^{58}$ Initial studies demonstrated that autophagy could impede early cancer development; however, high levels of autophagy in multiple cancers indicated that autophagy primarily promoted the progression of established cancers. ${ }^{59-62}$ Current research indicates that in cancer, autophagy can be neutral, tumor-suppressive, or tumor-promoting under different circumstances. Several researches have shown that autophagy is more likely to be used as a tumor suppressor. For example, monoallelic deletion of BECN1 (ATGO) occurred at high frequency in breast, ovarian, and prostate cancer; Beclin $1^{+/-}$ mice were tumor-prone; and overexpression of BECN1 inhibited tumor development. ${ }^{63-65}$ In agreement with these findings, studies of the deletion of other autophagy-regulating genes, such as Atgsand Bif-1, also provided evidence that autophagy performs tumor suppressive functions. ${ }^{66,67}$ Another crucial role of autophagy is chemoprevention by control of the level of selective autophagy cargo receptor p62/SOSTM. The accumulation of p62 has been observed in human tumors and is of the utmost importance in controlling tumor growth and oxidative stress. ${ }^{68}$ Deficiency of $p 62$ suppressed mammary, lung, and liver tumorigenesis induced by loss of $\operatorname{Atg} 7^{69,70}$ The relationship between defective autophagy and p62/SQSTM1 accumulation with tumorigenesis was also shown by the increased resistance of p62/SQSTM1 ${ }^{-1-}$ mice to Ras-induced lung carcinomas compared with wild-type animals, through a mechanism involving activation of the transcription factor NF- $\kappa \mathrm{B} .{ }^{71}$

In contrast, autophagy promotes the growth of advanced cancer within the tumor microenvironment. Degenhardt et al. ${ }^{72}$ first reported that autophagy was induced specifically in the hypoxic tumor regions, where it promotes tumor cell survival. In colorectal cancer (CRC), loss of the tumor suppressor Apc activates autophagy and tumorigenesis in the intestinal epithelium and Atg 7 deficiency prevents tumor initiation and progression with $A p c$ loss. These results indicate that autophagy promotes initiation and progression of intestinal cancer driven by Apcloss, suggesting the inhibition of autophagy could be considered for cancer prevention in individual susceptibility to $\mathrm{CRC}$. $^{73}$ A very recent study reported that autophagy was essential for tumor cell motility, as inhibition of autophagy blocks cell migration and invasion in vitro and reduces metastasis in vivo. Autophagy promoted focal adhesion (FA) disassembly through the interaction of processed LC3 with paxillin, a FA adaptor protein, and this interaction was promoted by phosphorylation of paxillin by oncogenic Src. This suggested that autophagy promoted tumor cell migration and metastasis. ${ }^{74}$ Overall, the studies described herein suggest that the role of autophagy in cancer depends on many factors including tissue type, tumor stage, and type of oncogenic mutation.

\section{Autophagy and cancer therapy}

Due to the contrasting context-dependent roles in tumor progression as discussed previously, a growing body of evidence implicates the biphasic role of autophagy following cancer therapeutics, whereby their anticancer potential may be enhanced or diminished. Conventional cancer therapies (e.g., cytotoxic drug and irradiation) have been shown to induce autophagy. Other anticancer drugs, like targeted therapy drugs (e.g., imatinib, cetuximab, bortezomib, vorinostat) and agents with different mechanisms of action (e.g., tamoxifen, ABT-737, nelfinavir), have also been shown to induce autophagy in tumor cells. Autophagy is stimulated as a protective mechanism to mediate the acquired resistance phenotype of some cancer cells against chemotherapy. In contrast, autophagy may act as an executioner by inducing autophagic cell death, a form of physiological non-apoptotic cell death. ${ }^{75}$ Based on the genetic and pharmacological studies reported to date, it appears that anticancer drugs result in different effects of autophagy on cell survival in different cancer 
types. $^{76}$

This is of particular significance because several cancer therapeutics, including tyrosine kinase inhibitors, mTOR inhibitors, ionizing radiation, microtubule inhibitors, and proteasome inhibitors, are known to modulate autophagy, which could interfere with tumor response. ${ }^{77}$ Currently, numerous chloroquine (CO) derivatives, including hydroxychloroquine (HCQ), quinacrine, mefloquine, and piperoquine, are used clinically. The lysosome inhibitor $\mathrm{CQ}$ is a 4-aminoquinoline that was originally used in the treatment or prevention of malaria. A number of the phase I/II clinical trials evaluated the maximum tolerated dose, safety, pharmacokinetics, and pharmacodynamics of HCQ in combination with other cancer therapies for the treatment of glioblastoma, advanced solid tumors, melanoma, and myeloma. ${ }^{78.82}$ Rangwala et al. ${ }^{81}$ reported that the combination of temsirolimus (an mTOR inhibitor) and HCQ improved the median progression-free survival in 13 melanoma patients to 3.5 months and increased the rate of stable disease in patients. In contrast to the sensitization of tumors to conventional chemotherapeutics by using the autophagy inhibitor $\mathrm{HCQ}$, the outcome from a recent phase I/II trial of HCQ in conjunction with radiation therapy and concurrent and adjuvant temozolomide, a DNA-damage agent, in patients with newly diagnosed glioblastoma multiforme showed no significant improvement in overall survival, which could be due to inconsistent inhibition of autophagy in patients treated with this regimen. ${ }^{83}$ There are concerns that neither $\mathrm{CQ}$ nor its derivatives are specific inhibitors of autophagy, and produce autophagy-independent cytotoxicity; this highlights the need for the development of more specific inhibitors targeting only autophagy.

In addition, the identification of novel targets, such as ULK1 kinase (the apical kinase important for initiating autophagosome formation) and Vps34 (the class III PI3K in the Beclin1 complex), and the development of small molecules targeting the components of the autophagy pathway other than lysosome inhibitors are currently receiving considerable attention. Thus, it is still necessary to conduct further clinical trials to investigate the benefits of chemotherapy in combination with autophagy inducers or inhibitors.

\section{Autophagy induction by apigenin}

Only a small number of studies have observed the induction of autophagy in response to apigenin and the results are controversial. ${ }^{8488}$ The effect of apigenin on autophagy in cancer cells was first reported by Ruela-de-Sousa et al. ${ }^{86}$ who showed that apigenin caused autophagy in TF1 erythroleukemia cells without triggering apoptosis. In TF1 cells, apigenin inhibited mTOR, an autophagy repressor, and its downstream target p70s6K. Interestingly, apigenin did not alter the level of Beclin 1, further reduced the levels of $\operatorname{Atg} 5,7$, and 12, and induced non-electron-dense vacuoles and double-membrane vacuoles, offering strong evidence of autophagy. These results suggested that autophagy is the dominant response to apigenin treatment in at least some types of leukemia. In addition, apigenin has been reported to exhibit autophagy-inducing effects in breast cancer T47D and MDA-MB-231 cells. ${ }^{84}$ Apigenin caused autophagy, which was evident from the appearance of autophagosomes, the accumulation of acidic vesicular organelles (AVOs), and the increase of LC3-II. Similarly, our recent study showed that apigenin induced both apoptosis and autophagy in HCT116 human colon cancer cells. ${ }^{85}$ In HCT116 cells, apigenin increased LC3-II protein levels, redistributed the protein LC3 into cytoplasmic puncta (a distinctive feature of autophagy), and increased the accumulation of AVO, indicating that the flavone may be effective for autophagosome formation. Apigenin treatment in C6 glioma cells resulted in the induction of both autophagy and apoptosis, however, autophagy occurred before apoptosis. The cells treated with $50 \mu \mathrm{mol} / \mathrm{L}$ apigenin showed a high rate of autophagic vacuole formation, and the cells treated with $100 \mu \mathrm{mol} / \mathrm{L}$ showed a high rate of cells in apoptosis. ${ }^{89} \mathrm{~A}$ recent study reported that apigenin could induce autophagic cell death in human thyroid cancer BCPAP cells, as evidenced by Beclin-1 accumulation, conversion of LC3 protein, p62 degradation, and significantly increased AVO formation. ${ }^{90}$ These results suggest that apigenin simultaneously induced apoptosis and autophagy.

Tong et al..$^{87}$ examined the effects of apigenin on AMPK modulation in human keratinocytes, and found that the induction of autophagy by apigenin-mediated AMPK activation was accompanied by inhibition of the MTOR signaling pathway, thereby suggesting that apigenin can act as a potent chemopreventive agent. In contrast, Jeremic et a $1 .{ }^{88}$ reported that apigenin caused autophagy in C6 glioma cells while apigenin-treated cells exhibited marked activation of mTOR accompanied by moderate activation of its upstream activator Akt and a decrease in AMPK phosphorylation; these effects suggested that the autophagy induced by apigenin in C6 glioma was AMPK/mTOR-independent.

\section{Effect of apigenin on crosstalk between apoptosis and autophagy}

Both the cellular self-destructive functions, apoptosis (self-ki- 
lling; type I PCD) and autophagy (self-eating; type II PCD) are well-controlled processes that play pivotal roles in development, tissue homeostasis, and disease. It is interesting that autophagy is often observed in dying cells. In many circumstances, this represents the cell's attempt to mitigate stress before resorting to the irreversible and final solution of apoptosis. Activation of autophagy, in some other settings, might reflect crosstalk between apoptosis and autophagy. Indeed, autophagy is induced by many stresses that may eventually lead to apoptosis, such as organelle dysfunction, metabolic stress, and pathogen infection. In general, cells restore autophagy to baseline levels and return to their initial state after the resolution of mild stress. However, if the stress is severe or persistent and autophagy fails to prolong cell survival, cells might respond by activating apoptosis in order to ensure a controlled and efficient elimination without triggering local inflammation. Therefore, it is plausible that interplay between autophagy and apoptosis conferred an evolutionary advantage to cells. Moreover, while autophagy and apoptosis are distinct processes with fundamentally different molecular features, several key inducers (e.g., Beclin-1, Bcl-2, Bcl-xL, mTOR, Atg5, p53) that regulate both processes had already been reported (Fig. 2) ${ }^{91-94}$ Further investigation is required to determine how the balance between apoptosis and autophagy is maintained in cancer cells.

During the past decade, more than 30 clinical trials have assessed the effect of autophagy manipulation on human cancer treatment (e.g., conventional or targeted agents), half of which used pharmacological inhibitors of autophagy such as CQ or HCQ. ${ }^{95.96}$ There is growing evidence to indicate that natural agents can modulate autophagy in cancer cells either positively or negatively. Therefore, the cytoprotective autophagy induced by natural products was always occurred in conjunction with apoptosis when this autophagy could be much more straightforward if the process was naturally regarded as cellular stress clearance. Cytoprotective autophagy is usually determined either by use of pharmacologic inhibitors of autophagy (such as CQ, bafilomycin A1, 3-methyladenine [3-MA], or ammonium chloride) or genetic silencing of autophagy-associated genes (such as Beclin-1, Atg 5, 7, or 12) to increase cancer cell sensitivity to autophagy-inducing stimuli via the promotion of apoptosis. Cao et al.${ }^{84}$ found that apigenin could induce both apoptosis and autophagy in breast cancer cells. In addition, the combination of apigenin with the autophagy inhibitor 3-MA was much more effective in the induction of apoptosis compared to apigenin alone. Another study from our group also reported similar results of a chemopreventive effect of apigenin against colon cancer. ${ }^{85}$
Apigenin induced both apoptosis and autophagy in HCT116 cells. Treatment with apigenin and 3-MA significantly increased the percentage of cells undergoing apoptosis and PARP-cleavage, which indicated that inhibition of autophagy significantly increased the percentage of cells undergoing apoptosis and PARP-cleavage. These results demonstrated that apigenin-mediated autophagy may play a cytoprotective role in apigenin-induced apoptosis. The aforementioned results support the evidence demonstrating that apigenin-induced autophagy is implicated in cell survival and contributes to the cytoprotective role of autophagy (Fig. 2).

An alternative cytoprotective pathway by apigenin-induced autophagy in cancer cells has also documented. The role of autophagy in cancer cells is dichotomous, with some reports demonstrating autophagy helps cells to survive under stress, and other reports indicating that autophagy ends in apoptosis in the absence of cell recovery. Zhang et al..$^{90}$ found that apigenin could induce both apoptosis and autophagy, while inhibition of

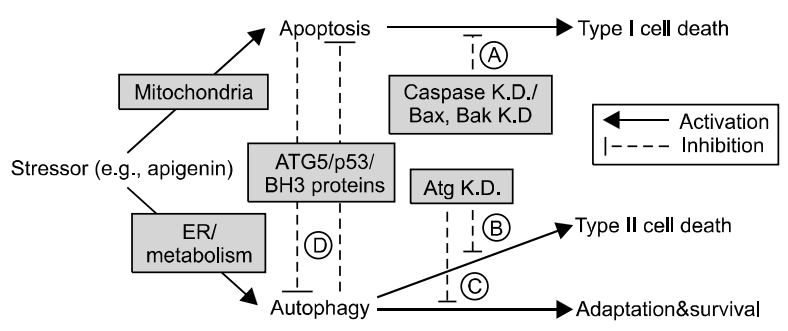

Figure 2. Signaling pathways and molecules involved in crosstalk between apoptosis and autophagy. Cellular stressors such as apigenin can start mitochondria outer membrane permeabilization and subsequent cytochrome $c$ release and apoptosis induction, while nutrient deficiency or ER stress can cause autophagy activation. Under physiological conditions, apoptosis and autophagy keep each other inactive through mutual inhibition. A strong apoptotic stimulus (for example DNA damage, death-receptor stimulation, or cytokine deprivation) can drive a cell into apoptotic 'type I' cell death. If apoptosis is inhibited under such conditions (by caspase knockout or Bax/Bak knockout, [A]), autophagy can become activated and result in a delayed 'type II' cell death through degradation of most cytoplasmic cell components and organelles. Under these circumstances, the K.D. of autophagy related genes $[B]$ reduces cell death. Autophagy can become activated through ER stress (for example, accumulation of misfolded proteins in the ER or intracellular calcium release from the ER) or nutrient deficiency. The cell then ensures survival by enhancing metabolic recycling through autophagy and adapting to the new nutrient conditions. K.D. of autophagy genes in such a situation leads to an increase in apoptotic 'type I' cell death [C]. The crosstalk between apoptosis and autophagy [D] is mediated via proteolytic processing of ATG5, the transcription factor p53, and the binding and subcellular localization of Bcl-2 family proteins with $\mathrm{BH} 3$ domains. Data from Jaeger and Wyss-Coray. ${ }^{92}$ ER, endoplasmic reticulum; K.D., knockdown. 
autophagy with the small molecule inhibitor 3-MA reduced cell death, indicating that apigenin was associated with autophagic cell death in BCPAP cells. Ruela-de-Sousa and et $a 1 .{ }^{86}$ investigated the molecular mode of action of apigenin and found that apigenin-induced autophagy was not followed by apoptosis in TF1 leukemia cells. When cells were exposed to a combination of apigenin and vincristine, a member of the vinca alkaloids family of chemotherapeutics, they became desensitized to vincristine-induced cell death. The influence of autophagy acquired by apigenin on cell death, particularly in the above mentioned cases, may be too complicated to explain currently, however, it may indicate that cytoprotective autophagy, as a decision-maker, may locate at the center of alternative courses of death or life of cancer cell fate.

Most of the recent studies have investigated autophagy induction by apigenin; however, only one study has considered the inhibitory property of apigenin on autophagy. Mohan et al. ${ }^{97}$ observed that apigenin suppressed starvation-induced autophagy in human malignant neuroblastoma cells. A combination of apigenin and the synthetic retinoid $\mathrm{N}$-(4-hydroxyphenyl) retinamide (4-HPR) synergistically reduced residual cell viability in the serum-starved neuroblastoma cells. While serum starvation increased the expression of LC3II in neuroblastoma SH-SY5Y cells, combination treatment effectively downregulated autophagy promoting proteins, including Beclin-1, LC3 II, Toll-like receptor-4, and myeloid differentiation primary response 88 , and activated the inhibitory autophagy signaling pathway $\mathrm{p}$-Akt/mTOR. It was concluded the combination of 4-HPR and apigenin worked synergistically to suppress autophagy and promote apoptosis in human neuroblastoma cells.

\section{CONCLUSIONS}

Here, we have discussed the beneficial roles of apigenin in the prevention and treatment of cancer through the induction of apoptosis and autophagy. Evidence from both in vitro and in vivo studies suggests that apigenin can trigger apoptosis and/or autophagy, which play pivotal roles in promotion and suppression of carcinogenesis. However, further in-depth investigations are needed to completely understand the mechanism of action and chemopreventive/therapeutic potential against human cancers. Interestingly, apigenin has been found to be bioavailable following oral administration in rats and mice, although no data are available for its pharmacokinetic and pharmacodynamic profiles in humans. The body of evidence concerning apigenin is fascinating and deserves greater atten- tion. As chemoprevention aims to stop the carcinogenic process or to prolong the onset of carcinogenesis by intervention with efficacious, non-toxic, and inexpensive agents to prevent, suppress, or reverse the malignant transformation, apigenin is one such agent that may satisfy most of these requirements. However, further information is required before apigenin can be brought to clinical trials, including data concerning the bioavailability and safety profile in human. Overall, the findings reported in the literature suggest that apigenin offers great potential for further investigation and development as a cancer chemopreventive and/or therapeutic agent.

\section{ACKNOWLEDGMENTS}

This work was supported by a 2-Year Research Grant of Pusan National University.

\section{CONFLICTS OF INTEREST}

No potential conflicts of interest were disclosed.

\section{REFERENCES}

1. Siegel RL, Miller KD, Jemal A. Cancer statistics, 2016. CA Cancer J Clin 2016:66:7-30.

2. Fidler MM, Soerjomataram I, Bray F. A global view on cancer incidence and national levels of the human development index. Int J Cancer 2016;139:2436-46.

3. Lippman SM, Hong WK. Cancer prevention science and practice. Cancer Res 2002;62:5119-25.

4. Wang X, Ouyang Y, Liu J, Zhu M, Zhao G, Bao W, et al. Fruit and vegetable consumption and mortality from all causes, cardiovascular disease, and cancer: systematic review and dose-response meta-analysis of prospective cohort studies. BMJ 2014:349:g4490.

5. Garcia-Closas R, Gonzalez CA, Agudo A, Riboli E. Intake of specific carotenoids and flavonoids and the risk of gastric cancer in Spain. Cancer Causes Control 1999;10:71-5.

6. Rossi M, Negri E, Talamini R, Bosetti C, Parpinel M, Gnagnarella $\mathrm{P}$, et al. Flavonoids and colorectal cancer in Italy. Cancer Epidemiol Biomarkers Prev 2006;15:1555-8.

7. Bosetti C, Spertini L, Parpinel M, Gnagnarella P, Lagiou P, Negri E, et al. Flavonoids and breast cancer risk in Italy. Cancer Epidemiol Biomarkers Prev 2005;14:805-8.

8. Gates MA, Vitonis AF, Tworoger SS, Rosner B, Titus-Ernstoff L, Hankinson SE, et al. Flavonoid intake and ovarian cancer risk in a population-based case-control study. Int J Cancer 2009;124:1918-25.

9. Horn-Ross PL, John EM, Canchola AJ, Stewart SL, Lee MM. Phytoestrogen intake and endometrial cancer risk. J Natl Cancer Inst 2003;95:1158-64.

10. Frankenfeld CL, Cerhan JR, Cozen W, Davis S, Schenk M, Morton LM, et al. Dietary flavonoid intake and non-Hodgkin lymphoma risk. Am J Clin Nutr 2008;87:1439-45. 
11. Rusznyák ST, Szent-Györgyi A. Vitamin P: flavonols as vitamins. Nature 1936;138:27.

12. Beecher GR. Overview of dietary flavonoids: nomenclature, occurrence and intake. J Nutr 2003;133:3248S-54S.

13. Birt DF, Walker B, Tibbels MG, Bresnick E. Anti-mutagenesis and anti-promotion by apigenin, robinetin and indole-3-carbinol. Carcinogenesis 1986;7:959-63.

14. Shukla S, Bhaskaran N, Babcook MA, Fu P, Maclennan GT, Gupta $\mathrm{S}$. Apigenin inhibits prostate cancer progression in TRAMP mice via targeting PI3K/Akt/FoxO pathway. Carcinogenesis 2014;35:452-60.

15. Shukla S, MacLennan GT, Fu P, Gupta S. Apigenin attenuates insulin-like growth factor-I signaling in an autochthonous mouse prostate cancer model. Pharm Res 2012;29:1506-17.

16. Silvan S, Manoharan S. Apigenin prevents deregulation in the expression pattern of cell-proliferative, apoptotic, inflammatory and angiogenic markers during 7,12-dimethylbenz[a]anthracene-induced hamster buccal pouch carcinogenesis. Arch Oral Biol 2013:58:94-101.

17. Silvan S, Manoharan S, Baskaran N, Anusuya C, Karthikeyan S, Prabhakar MM. Chemopreventive potential of apigenin in 7,12-dimethylbenz(a)anthracene induced experimental oral carcinogenesis. Eur J Pharmacol 2011;670:571-7.

18. Gómez-García FJ, López-Jornet MP, Alvarez-Sánchez N, Castillo-Sánchez J, Benavente-García O, Vicente Ortega V. Effect of the phenolic compounds apigenin and carnosic acid on oral carcinogenesis in hamster induced by DMBA. Oral Dis 2013;19:279-86.

19. Baldasquin-Caceres B, Gomez-Garcia FJ, López-Jornet P, Castillo-Sanchez J, Vicente-Ortega V. Chemopreventive potential of phenolic compounds in oral carcinogenesis. Arch Oral Biol 2014:59:1101-7.

20. Wei H, Tye L, Bresnick E, Birt DF. Inhibitory effect of apigenin, a plant flavonoid, on epidermal ornithine decarboxylase and skin tumor promotion in mice. Cancer Res 1990;50:499-502.

21. Byun S, Park J, Lee E, Lim S, Yu JG, Lee SJ, et al. Src kinase is a direct target of apigenin against UVB-induced skin inflammation. Carcinogenesis 2013;34:397-405.

22. Tong X, Mirzoeva S, Veliceasa D, Bridgeman BB, Fitchev $\mathrm{P}$, Cornwell ML, et al. Chemopreventive apigenin controls UVB-induced cutaneous proliferation and angiogenesis through HuR and thrombospondin-1. Oncotarget 2014:5:11413-27.

23. Bridgeman BB, Wang $\mathrm{P}$, Ye B, Pelling JC, Volpert OV, Tong X. Inhibition of mTOR by apigenin in UVB-irradiated keratinocytes: A new implication of skin cancer prevention. Cell Signal 2016;28:460-8.

24. Leonardi T, Vanamala J, Taddeo SS, Davidson LA, Murphy ME, Patil BS, et al. Apigenin and naringenin suppress colon carcinogenesis through the aberrant crypt stage in azoxymethane-treated rats. Exp Biol Med (Maywood) 2010;235:710-7.

25. Tatsuta A, Iishi H, Baba M, Yano H, Murata K, Mukai M, et al. Suppression by apigenin of peritoneal metastasis of intestinal adenocarcinomas induced by azoxymethane in Wistar rats. Clin Exp Metastasis 2000;18:657-62.

26. Zhong Y, Krisanapun C, Lee SH, Nualsanit T, Sams C, Peungvicha P, et al. Molecular targets of apigenin in colorectal cancer cells: involvement of p21, NAG-1 and p53. Eur J Cancer 2010;46:3365-74.

27. Shukla S, Fu P, Gupta S. Apigenin induces apoptosis by targeting inhibitor of apoptosis proteins and Ku70-Bax interaction in prostate cancer. Apoptosis 2014;19:883-94.

28. Pandey M, Kaur P, Shukla S, Abbas A, Fu P, Gupta S. Plant flavone apigenin inhibits HDAC and remodels chromatin to induce growth arrest and apoptosis in human prostate cancer cells: in vitro and in vivo study. Mol Carcinog 2012;51:952-62.

29. Shukla S, Mishra A, Fu P, MacLennan GT, Resnick MI, Gupta S. Up-regulation of insulin-like growth factor binding protein-3 by apigenin leads to growth inhibition and apoptosis of $22 \mathrm{Rv} 1$ xenograft in athymic nude mice. FASEB J 2005;19:2042-4.

30. Shukla S, Kanwal R, Shankar E, Datt M, Chance MR, Fu P, et al. Apigenin blocks IKK $\alpha$ activation and suppresses prostate cancer progression. Oncotarget 2015;6:31216-32.

31. Shukla S, Gupta S. Molecular targets for apigenin-induced cell cycle arrest and apoptosis in prostate cancer cell xenograft. Mol Cancer Ther 2006;5:843-52.

32. Shukla S, Gupta S. Apigenin-induced prostate cancer cell death is initiated by reactive oxygen species and p53 activation. Free Radic Biol Med 2008;44:1833-45.

33. Mafuvadze B, Liang Y, Besch-Williford C, Zhang X, Hyder SM. Apigenin induces apoptosis and blocks growth of medroxyprogesterone acetate-dependent BT-474 xenograft tumors. Horm Cancer 2012;3:160-71.

34. Shao H, Jing K, Mahmoud E, Huang H, Fang X, Yu C. Apigenin sensitizes colon cancer cells to antitumor activity of ABT-263. Mol Cancer Ther 2013;12:2640-50.

35. Wang $\mathrm{QR}$, Yao XQ, Wen G, Fan Q, Li YJ, Fu XQ, et al. Apigenin suppresses the growth of colorectal cancer xenografts via phosphorylation and up-regulated FADD expression. Oncol Lett 2011:2:43-7.

36. Chunhua L, Donglan L, Xiuqiong F, Lihua Z, Qin F, Yawei L, et al. Apigenin up-regulates transgelin and inhibits invasion and migration of colorectal cancer through decreased phosphorylation of AKT. J Nutr Biochem 2013;24:1766-75.

37. Liu LZ, Fang J, Zhou Q, Hu X, Shi X, Jiang BH. Apigenin inhibits expression of vascular endothelial growth factor and angiogenesis in human lung cancer cells: implication of chemoprevention of lung cancer. Mol Pharmacol 2005:68:635-43.

38. King JC, Lu QY, Li G, Moro A, Takahashi H, Chen M, et al. Evidence for activation of mutated p53 by apigenin in human pancreatic cancer. Biochim Biophys Acta 2012;1823:593-604.

39. Johnstone RW, Ruefli AA, Lowe SW. Apoptosis: a link between cancer genetics and chemotherapy. Cell 2002;108:153-64.

40. Hanahan D, Weinberg RA. Hallmarks of cancer: the next generation. Cell 2011;144:646-74.

41. Ashkenazi A. Targeting the extrinsic apoptosis pathway in cancer. Cytokine Growth Factor Rev 2008;19:325-31.

42. Green DR, Kroemer G. The pathophysiology of mitochondrial cell death. Science 2004:305:626-9.

43. Masuelli L, Marzocchella L, Quaranta A, Palumbo C, Pompa G, Izzi $\mathrm{V}$, et al. Apigenin induces apoptosis and impairs head and neck carcinomas EGFR/ErbB2 signaling. Front Biosci (Landmark Ed) $2011 ; 16: 1060-8$.

44. Zhang $\mathrm{Q}$, Zhao XH, Wang ZJ. Flavones and flavonols exert cytotoxic effects on a human oesophageal adenocarcinoma cell line (OE33) by causing G2/M arrest and inducing apoptosis. Food Chem Toxicol 2008:46:2042-53.

45. Zhang Q, Zhao XH, Wang Z). Cytotoxicity of flavones and flavonols to a human esophageal squamous cell carcinoma cell line (KYSE-510) by induction of G2/M arrest and apoptosis. Toxicol In Vitro 2009:23:797-807.

46. Turktekin M, Konac E, Onen HI, Alp E, Yilmaz A, Menevse S. 
Evaluation of the effects of the flavonoid apigenin on apoptotic pathway gene expression on the colon cancer cell line (HT29). J Med Food 2011;14:1107-17.

47. Izeradjene K, Douglas L, Delaney A, Houghton JA. Casein kinase II (CK2) enhances death-inducing signaling complex (DISC) activity in TRAIL-induced apoptosis in human colon carcinoma cell lines. Oncogene 2005:24:2050-8.

48. Farah M, Parhar K, Moussavi M, Eivemark S, Salh B. 5,6-Dichloro-ribifuranosylbenzimidazole- and apigenin-induced sensitization of colon cancer cells to TNF-alpha-mediated apoptosis. Am J Physiol Gastrointest Liver Physiol 2003;285:G919-28.

49. Khan TH, Sultana S. Apigenin induces apoptosis in Hep G2 cells: possible role of TNF-alpha and IFN-gamma. Toxicology 2006;217: 206-12.

50. Kim BR, Jeon YK, Nam MJ. A mechanism of apigenin-induced apoptosis is potentially related to anti-angiogenesis and anti-migration in human hepatocellular carcinoma cells. Food Chem Toxicol 2011;49:1626-32.

51. Lee SH, Ryu JK, Lee KY, Woo SM, Park JK, Yoo JW, et al. Enhanced anti-tumor effect of combination therapy with gemcitabine and apigenin in pancreatic cancer. Cancer Lett 2008;259:39-49.

52. De Duve C, Wattiaux R. Functions of lysosomes. Annu Rev Physiol 1966;28:435-92.

53. Jardon MA, Rothe K, Bortnik S, Vezenkov L, Jiang X, Young RN, et al. Autophagy: from structure to metabolism to therapeutic regulation. Autophagy 2013;9:2180-2.

54. Rubinsztein DC, Codogno P, Levine B. Autophagy modulation as a potential therapeutic target for diverse diseases. Nat Rev Drug Discov 2012;11:709-30.

55. Kroemer G, Mariño G, Levine B. Autophagy and the integrated stress response. Mol Cell 2010;40:280-93.

56. Ravikumar B, Futter M, Jahreiss L, Korolchuk VI, Lichtenberg M, Luo S, et al. Mammalian macroautophagy at a glance. J Cell Sci 2009;122:1707-11.

57. Kaushik S, Bandyopadhyay U, Sridhar S, Kiffin R, Martinez-Vicente $\mathrm{M}$, Kon $\mathrm{M}$, et al. Chaperone-mediated autophagy at a glance. J Cell Sci 2011;124:495-9.

58. White E. Deconvoluting the context-dependent role for autophagy in cancer. Nat Rev Cancer 2012;12:401-10.

59. Lorin S, Hamaï A, Mehrpour M, Codogno P. Autophagy regulation and its role in cancer. Semin Cancer Biol 2013;23:361-79.

60. Rosenfeldt MT, O'Prey J, Morton JP, Nixon C, MacKay G, Mrowinska A, et al. p53 status determines the role of autophagy in pancreatic tumour development. Nature 2013;504:296-300.

61. Parkhitko A, Myachina F, Morrison TA, Hindi KM, Auricchio N, Karbowniczek M, et al. Tumorigenesis in tuberous sclerosis complex is autophagy and p62/sequestosome 1 (SQSTM1)-dependent. Proc Natl Acad Sci U S A 2011;108:12455-60.

62. Rao S, Tortola L, Perlot T, Wirnsberger G, Novatchkova M, Nitsch $\mathrm{R}$, et al. A dual role for autophagy in a murine model of lung cancer. Nat Commun 2014:5:3056.

63. Qu X, Yu J, Bhagat G, Furuya N, Hibshoosh H, Troxel A, et al. Promotion of tumorigenesis by heterozygous disruption of the beclin 1 autophagy gene. J Clin Invest 2003;112:1809-20.

64. Karantza-Wadsworth V, White E. Role of autophagy in breast cancer. Autophagy 2007:3:610-3.

65. Liang XH, Jackson S, Seaman M, Brown K, Kempkes B, Hibshoosh $\mathrm{H}$, et al. Induction of autophagy and inhibition of tumorigenesis by beclin 1. Nature 1999;402:672-6.
66. Takahashi Y, Coppola D, Matsushita N, Cualing HD, Sun M, Sato Y, et al. Bif-1 interacts with Beclin 1 through UVRAG and regulates autophagy and tumorigenesis. Nat Cell Biol 2007;9:1142-51.

67. Mariño G, Salvador-Montoliu N, Fueyo A, Knecht E, Mizushima N, López-Otín C. Tissue-specific autophagy alterations and increased tumorigenesis in mice deficient in Atg4C/autophagin-3. J Biol Chem 2007;282:18573-83.

68. Moscat J, Diaz-Meco MT. p62 at the crossroads of autophagy, apoptosis, and cancer. Cell 2009;137:1001-4.

69. Moscat J, Diaz-Meco MT. p62: a versatile multitasker takes on cancer. Trends Biochem Sci 2012;37:230-6.

70. Wei H, Wang C, Croce CM, Guan JL. p62/SQSTM1 synergizes with autophagy for tumor growth in vivo. Genes Dev 2014:28:1204-16.

71. Duran A, Linares JF, Galvez AS, Wikenheiser K, Flores JM, Diaz-Meco MT, et al. The signaling adaptor p62 is an important NF-kappaB mediator in tumorigenesis. Cancer Cell 2008;13:343-54.

72. Degenhardt K, Mathew R, Beaudoin B, Bray K, Anderson D, Chen G, et al. Autophagy promotes tumor cell survival and restricts necrosis, inflammation, and tumorigenesis. Cancer Cell 2006;10:51-64.

73. Lévy J, Cacheux W, Bara MA, L'Hermitte A, Lepage P, Fraudeau M, et al. Intestinal inhibition of Atg7 prevents tumour initiation through a microbiome-influenced immune response and suppresses tumour growth. Nat Cell Biol 2015:17:1062-73.

74. Sharifi MN, Mowers EE, Drake LE, Collier C, Chen H, Zamora M, et al. Autophagy promotes focal adhesion disassembly and cell motility of metastatic tumor cells through the direct interaction of paxillin with LC3. Cell Rep 2016;15:1660-72.

75. Bincoletto C, Bechara A, Pereira GJ, Santos CP, Antunes F, Peixoto da-Silva J, et al. Interplay between apoptosis and autophagy, a challenging puzzle: new perspectives on antitumor chemotherapies. Chem Biol Interact 2013;206:279-88.

76. Yang ZJ, Chee CE, Huang S, Sinicrope FA. The role of autophagy in cancer: therapeutic implications. Mol Cancer Ther 2011;10:1533-41.

77. Benbrook DM, Long A. Integration of autophagy, proteasomal degradation, unfolded protein response and apoptosis. Exp Oncol 2012;34:286-97.

78. Mahalingam D, Mita M, Sarantopoulos J, Wood L, Amaravadi RK, Davis LE, et al. Combined autophagy and HDAC inhibition: a phase I safety, tolerability, pharmacokinetic, and pharmacodynamic analysis of hydroxychloroquine in combination with the HDAC inhibitor vorinostat in patients with advanced solid tumors. Autophagy 2014;10:1403-14.

79. Vogl DT, Stadtmauer EA, Tan KS, Heitjan DF, Davis LE, Pontiggia L, et al. Combined autophagy and proteasome inhibition: a phase 1 trial of hydroxychloroquine and bortezomib in patients with relapsed/refractory myeloma. Autophagy 2014:10:1380-90.

80. Rangwala R, Chang YC, Hu J, Algazy KM, Evans TL, Fecher LA, et al. Combined MTOR and autophagy inhibition: phase I trial of hydroxychloroquine and temsirolimus in patients with advanced solid tumors and melanoma. Autophagy 2014;10:1391-402.

81. Rangwala R, Leone R, Chang YC, Fecher LA, Schuchter LM, Kramer A, et al. Phase I trial of hydroxychloroquine with dose-intense temozolomide in patients with advanced solid tumors and melanoma. Autophagy 2014;10:1369-79.

82. Yang A, Rajeshkumar NV, Wang X, Yabuuchi S, Alexander BM, Chu GC, et al. Autophagy is critical for pancreatic tumor growth and progression in tumors with p53 alterations. Cancer Discov 2014:4:905-13.

83. Rosenfeld MR, Ye X, Supko JG, Desideri S, Grossman SA, Brem S, 
et al. A phase I/II trial of hydroxychloroquine in conjunction with radiation therapy and concurrent and adjuvant temozolomide in patients with newly diagnosed glioblastoma multiforme. Autophagy 2014;10:1359-68.

84. Cao X, Liu B, Cao W, Zhang W, Zhang F, Zhao H, et al. Autophagy inhibition enhances apigenin-induced apoptosis in human breast cancer cells. Chin J Cancer Res 2013;25:212-22.

85. Lee Y, Sung B, Kang YJ, Kim DH, Jang JY, Hwang SY, et al. Apigenin-induced apoptosis is enhanced by inhibition of autophagy formation in HCT116 human colon cancer cells. Int J Oncol 2014:44:1599-606

86. Ruela-de-Sousa RR, Fuhler GM, Blom N, Ferreira CV, Aoyama H, Peppelenbosch MP. Cytotoxicity of apigenin on leukemia cell lines: implications for prevention and therapy. Cell Death Dis 2010;1:e19.

87. Tong X, Smith KA, Pelling JC. Apigenin, a chemopreventive bioflavonoid, induces AMP-activated protein kinase activation in human keratinocytes. Mol Carcinog 2012;51:268-79.

88. Jeremic I, Tadic V, Isakovic A, Trajkovic V, Markovic I, Redzic Z, et al. The mechanisms of in vitro cytotoxicity of mountain tea, Sideritis scardica, against the C6 glioma cell line. Planta Med 2013;79:1516-24.

89. Coelho PL, Oliveira MN, da Silva AB, Pitanga BP, Silva VD, Faria GP, et al. The flavonoid apigenin from Croton betulaster Mull inhibits proliferation, induces differentiation and regulates the in- flammatory profile of glioma cells. Anticancer Drugs 2016;27:960-9.

90. Zhang L, Cheng X, Gao Y, Zheng J, Xu Q, Sun Y, et al. Apigenin induces autophagic cell death in human papillary thyroid carcinoma BCPAP cells. Food Funct 2015;6:3464-72.

91. Maiuri MC, Zalckvar E, Kimchi A, Kroemer G. Self-eating and self-killing: crosstalk between autophagy and apoptosis. Nat Rev Mol Cell Biol 2007;8:741-52.

92. Jaeger PA, Wyss-Coray T. All-you-can-eat: autophagy in neurodegeneration and neuroprotection. Mol Neurodegener 2009;4:16.

93. El-Khattouti A, Selimovic D, Haikel Y, Hassan M. Crosstalk between apoptosis and autophagy: molecular mechanisms and therapeutic strategies in cancer. J Cell Death 2013;6:37-55.

94. Booth LA, Tavallai S, Hamed HA, Cruickshanks N, Dent P. The role of cell signalling in the crosstalk between autophagy and apoptosis. Cell Signal 2014:26:549-55.

95. Maes H, Rubio N, Garg AD, Agostinis P. Autophagy: shaping the tumor microenvironment and therapeutic response. Trends Mol Med 2013;19:428-46.

96. Jiang P, Mizushima N. Autophagy and human diseases. Cell Res 2014:24:69-79.

97. Mohan N, Banik NL, Ray SK. Combination of N-(4-hydroxyphenyl) retinamide and apigenin suppressed starvation-induced autophagy and promoted apoptosis in malignant neuroblastoma cells. Neurosci Lett 2011:502:24-9. 